\title{
De pressiouibus negativis in sinu et in pariete regionis fluido viscoso moventi impletae schedula.
}

C. TruespeuL (Baltimora) $\left(^{*}\right)$

A Bruno Finai nel 70 no compleanno.

Sunto. - Si dimostrano condizioni necessarie e sufficienti affuchè la pressione su un elemento generico in un fluido viscoso sia nonnegativa. Il criterio si esprime mediante il "numero di tensione".

\section{1. - De sensu et momento pressionis uegativae.}

Late creditur fluidum in statu aequilibrii pressionem negativam tollendi non esse capax. Utrum ita sit in fluido etiam moventi, an pressio negativa in aliquibus punctis tempora saltem satis brevia accidere queat, adhue incertum manere videtur. In tractatibus classicis de theoria fluidorum perfectorum nullius compressionis capacium, ille valor constantis pressionis $p_{0}$ quae in determinationem problematis particularis finibus et velocitatibus incohantibus datis determinati init, qui sit minimus at dentur locus tempusque quo pressio $p$ evanescat, ad indicium principii separationis fluidi ab pariete aut cavitationis in sinu fluidi ipsius accipitur ( $\left.{ }^{1}\right)$. Contra autem res se habet in theoria gasorum perfectorum ubi ab initio postnlari solet tali modo $p=p(\rho, \theta)$ pres. sionem staticam subici, $\rho$ densitate et $\theta$ temperatura, ut ex eo sequatur semper et ubique limes $p \geqq 0$. Secundum igitar notiones, quae intrinsecus in theoriis fluidorum perfectorum insunt, cavitatio et capacitas dilatandi se mutuo exclu. dunt. Tamen in theoria fluidoram viscosorum, quae regulas generaliores illis quae ex ambobus theoriis fluidorum perfectorum eliciendae sunt praebere deberet, adbue (qua invenio) tota haec materia indagatione caret, quod argumentum harum mearum observationum simplicium statuit.

De eavitatione in fluidis naturalibus hic sermo non est. An theoremata mathematica quae sequentar utilia in re physica probabuntur, decet physicis relinquere.

Ad analysin intelligendam recordari juvabit, quod pressio normalis, quam

(*) Entrata in Redazione il 26 gennaio 1970.

(1) V. ex. gr. $\$ 86$ commentationis EuLERI, Sectio secrnda de principiis motus fluidorum, Novi comm. acad. sci. Petrop. 14 (1769): I, 270386 (1770)=Opera omnia (II) 13, 73-158, cuius sententia ab innumerabilibus auctoribus posteris resonata est. 
in fluido viscoso moventi superficies aut introrsum ant in pariete patitur, multo a pressione statica $p(\rho, \theta)$ quae densitati temperaturaeque in eodem fluido si sit quietum conveniat, discrepare potest.

\section{2. - Numerus tractilis in gaso viscoso compressionis capaei.}

Detur gasum viscosum, ouius tensor conatuum ( $\left.{ }^{2}\right) \boldsymbol{T}$ ex pressione statica $p$ atque tensore tractuum $\left({ }^{3}\right) D$ necnon viscositatibus $\lambda$ ef $\mu$ regula constitutionis Navier-Stokesiana determinatur:

$$
T=(-p+\lambda \bar{\delta}) 1+2 \mu D
$$

ubi $\delta \equiv \operatorname{tr} D=\operatorname{div} \dot{x}$ atque $p, \lambda$ et $\mu$ functiones datae densitatis $\rho$ et temperaturae $\theta$ quae inaequalitatibus sequentibus subiciuntur:

$$
p \geqq 0, \quad \lambda+\frac{2}{3} \mu \geqq 0, \quad \mu \geqq 0 .
$$

Postea seponens in hac re locos singulares et fluida perfecta specialiave in quibus $p=0$ aut $\mu=0$, assumo esse semper et ubique

$$
p>0 \text { et } \mu>0 \text {. }
$$

Appelletur numerus amplitudiniviscosus ratio nondimensionalis $\mathfrak{H}$ viscositatis voluminis $3 \lambda+2 \mu$ ad viseositatem lapsus $\mu$, id est

$$
\mathfrak{H B} \equiv 1+\frac{3 \lambda}{2 \mu} .
$$

Ex Aeq. $(2)_{1}$ et Inaeq. $(3)_{2}$ sequitur, ut sit

$$
\infty>\mathfrak{H} \geqq 0
$$

Si $\mathfrak{B}=0$, dicitur fluidum «modo STokesiano

Tractio $\boldsymbol{t}$, quae in elementum orientatam superficiei cuias versor $n$ est exseritur, formula

$$
t=(-p+\lambda \delta) n+2 \mu D n
$$

$\left(^{2}\right)$ Italice «tensore degli sforzi».

(3) Italice «tensore di velocità di deformaziono", id est

$$
2 D=\operatorname{grad} \dot{x}+(\operatorname{grad} \dot{x})^{T},
$$

whi $\dot{x}$ campus velooitatum. 
datur, ex qua invenitur pressio normalis $p_{(\boldsymbol{n})}$ quam patitur elementum:

$$
\begin{aligned}
p_{(\boldsymbol{n})} & =-\boldsymbol{t} \cdot n, \\
& =p-\lambda \delta-2 \mu \boldsymbol{n} \cdot \boldsymbol{D} \boldsymbol{n}, \\
& =p-\lambda\left(D_{\lambda}+D_{2}+D_{3}\right)-2 \mu D_{(n)},
\end{aligned}
$$

ubi $D_{1}, D_{2}, D_{3}$ tractus principales et $D_{(n)}$ tractus secundum directionem cuius versor $n$.

Ut inter omnia elementa superficiei in dato puncto illud inveniamus, quod pressionem normalem minimam sustineat, eligimus talem $\mathbf{n}$, qualis tractum $D_{(n)}$ maximum reddat, et hic $n$ versor directionis principalis tensoris tractuum $D$ est, qui autovalori maximo convenit. Si tractus principales $D_{i}$ ordinamus ut sit $D_{1} \geqq D_{2} \geqq D_{3}$, est igitur nax $D_{(n)}=D_{1}$, at reperiatur

$$
\min p_{(\boldsymbol{n})}=p-\lambda\left(D_{1}+D_{2}+D_{3}\right)-2 \mu D_{1} \text {. }
$$

Si $\min p_{(n)} \geqq 0$, sequitur ut sit $p_{(n)} \geqq 0$ versore $n$ quocunque.

Ut analyseos summam faciamus adjuvat numerum tractilem Te nondimensionalem definire:

$$
\widetilde{T} \equiv \frac{2 \mu}{3 p}\left[\left(D_{1}-D_{2}\right)+\left(D_{1}-D_{3}\right)+\mathfrak{J}\left(D_{1}+D_{2}+D_{3}\right)\right] .
$$

Ex superioribus sequitur

Theorema. - Datis in puncto $x$ valoribus $\rho$ et $\theta$, ut nullum elementum superficiei ibi pressionem normalem negativam patiatur, non solum sufficit sed eliam necesse est, ut sit

$$
\text { Te }<1 \text {. }
$$

Si autem $\mathbb{T e}=1$, evanescit pressio normalis quae in elementum exseritur, cuius versor axi principali qui tractui maximo convenit, parallelus est.

Definitione numeri Te perpensa, colliguntur causae, quae pressiones normales negativas in fluidis viscosis efficere valeant:

10. - Magna distractio, id est, aut $D_{1}>>D_{2}$ aut $D_{2}>>D_{3}$ aut ambo inaequalitates obtineunt.

$2^{\circ}$. - Numero amplitudiniviscoso $\mathfrak{B}$ non nullo, magna dilatatio positiva, id est, $D_{1}+D_{2}+D_{3}>>0$. Si autem $\mathfrak{J B}=0$, sola causa prior efficere potest.

\section{3. - Applicationes in sinu fluidi.}

In dilatatione pura $D_{1}=D_{2}=D_{3}=D=\frac{1}{3} \delta$ et hinc

$$
\tau_{e}=\frac{2 \mu \mathbb{B} D}{p} .
$$


In compressione $D<0$; sequitur ut sit $\mathbb{T e} \leqq 0$ et a fortiori $\mathbb{T e}<1$; in omni igitur compressione pura pressiones negativae ant nullae absunt. In expansione antem casus duo discernendi sunt. $1^{0}$, si valeat modus STokesianus, sequitur ut sit $T e=0$, et pressio normalis pressioni staticae aequalis est (quod etiam in compressione valet). Hoo in casu sequitur denuo a fortiori pressiones ne. gativas aut nullas abesse. $2^{\circ}$, si $\mathfrak{H B}>0$ et si

$$
D>\frac{p}{2 \mu \mathrm{IB}}
$$

pressio normalis negativa erit, et videtur fluidum embolum retrorsum trabere, quod expansionem producere conaretur. Utrum talis effectus in praxi contin. gat, an fluidum embolum non sequi possit, at spatium vacuum inter se et embolum reliquat, oportet ex serutatoribus in re aerodynamica petere.

(In theoria cinetica gasorum tales definitiones finguntur, ut omnes pressiones normales affirmativas reddant. Non solum igitur possibilitas cavitationis aut separationis modo allata in ista theoria non datur, sed etiam ipsa theoria cum $\mathrm{N}_{\mathrm{AVIER}}-\mathrm{S}_{\mathrm{T}} \mathrm{k}$ esiana in casibus quadrare nequit ubi ex hac pressio negativa elicitur. Hanc discrepantiam alibi iam exposui et indagavi ( $\left.{ }^{4}\right)$.

In motu isochorico $\delta=0$ et hinc

$$
\tau e=\frac{2 \mu D_{1}}{p}
$$

Numerus Te igitur a solo tractu maximo pendet, et $\mathbb{T e} \geqq 0$.

Generalius, sit $\mathbb{C} q_{0}$ numerus tractilis qui fluido et motui conveniat, si modus Sromxsianus valeat:

$$
\widetilde{\widetilde{C}} \mathfrak{C}_{0} \equiv \frac{2 \mu}{3 p}\left[\left(D_{1}-D_{2}\right)+\left(D_{1}-D_{3}\right)\right]
$$

Semper $\widetilde{T} e_{0} \geqq 0$, et

$$
\mathbb{T e}=\mathfrak{C e}_{0}+\frac{2 \mu \mathrm{JB} \delta}{3 p}
$$

Si fluidum se dilatat, $\mathbb{T e}_{e} \geqq \mathbb{T e}_{0}$, sed si se contrahit, $\mathbb{T e} \leqq \mathbb{T} e_{0}$. In motu astringendi igitur sufficit inaequalitas $\mathbb{T}_{0}<1$ ut nulla pressio normalis negativa eliciatur.

In indagationibus theoriarum fluidorum nonlinearium reperitur numerus truncationis (5), qui formula

$$
\mathbb{T} \mathfrak{l}=\frac{\sqrt{2} \mu \sqrt{D_{1}^{2}+D_{2}^{2}+D_{3}^{2}}}{p}
$$

(') V. schedulam A precise upper limit for the correctness of the Navier.Stokes theory with respect to the kinetic theory, J. Statistical Physies 1, 313-318 (1969).

(5) Italice: numero di troncatura. V. ex. gr. schedulam meam, On the differential equations of slip flow, Proc. Nat. Acad. Sci. U.S.A. 34, 342-347 (1948). 
definirı potest. Hic numerus nondimensionalis indiciam praebet an in theoriis simplieioribus de fluidis nonlinenribus potentiae tensoris $D$ prima superiores in regula constitutionis tuto negligi possunt. Si $\mathbb{C} \mathfrak{r}<<1$, licet dicere tractus «minimos» esse. Ex Aeqq. (16) et (13) deducitur in motu eidem plano parallelo et isochorico, cum sint $D_{2}=0$ et $D_{3}=-D_{1}$, esse

$$
\tau e=\tau r .
$$

Generalius valet inaequalitas non difficilis demonstratu:

$$
\mathbb{C} \mathfrak{e} \leqq \frac{2}{\sqrt{3}} \sqrt{1+\frac{1}{2} \mathfrak{B B}^{2}} \mathbb{T} \mathfrak{r}
$$

ubi limes superior optimus est, quandoquidem valoribus sequentibus substitutis,

$$
D_{2}=D_{3}=\frac{\mathfrak{B}-1}{\mathfrak{B}+2} D_{1}
$$

assumpto numero $D_{1} \geqq 0$, invenietur aequalitatem contingere.

Ex Inaeqq. (18) et (10) intelligitur, in motu fluidi ubi tractus satis parvi sunt pressionem normalem negativam numquam dari. Hane in finem sufficit ut sit

$$
\tau_{\mathfrak{r}}<\frac{\sqrt{3}}{2 \sqrt{1+\frac{1}{2} \mathfrak{B}^{2}}}
$$

at huic condicioni necessitas multo deest, uti videmus ex exemplo dilatationis purae fluidi, quod modo Stozesiano obtemperat, quoniam $\tau e=0$ sed $\tau_{\mathfrak{r}}=$ $=\sqrt{6} \mu D / p$, ex quibus patet pressionem negativam non exsistere, quamvis magnus sit numerus $\mathbb{C}$.

\section{4. - Digressio de cinematica parietis in quiete.}

Posito fluidum in pariete quieto adhaerere, at in eo sit $\dot{x}=\mathbf{0}$, gradiens velocitatum ibi formam simplicissimam assumit. Ut valgo scitur, axis circumactuum parietem tangit. Sit $W$ tensor circumactuum ( $\left.{ }^{6}\right)$, sit e versor eius axis, sit $n$ versor superficiei, et eligatur $f$ ut sit $(n, e, f)$ basis orthonormalis vectorum

(6) Italice a tensore del vortice», id est

$$
2 \boldsymbol{W}=\operatorname{grad} \dot{x}-(\operatorname{grad} \dot{x})^{T},
$$


in puneto superficiei. His positis,

$$
W=\frac{1}{2} w_{n} \wedge f, \quad W n=-\frac{1}{2} w f, \quad W f=+\frac{1}{2} w_{n},
$$

ubi $w$, qui est numerus nonnegativas, vorticitas appellatur. Ex theoremate Ef. Horati AgNi ( ${ }^{7}$ ) pro calculatione derivatae elementi superficiei invenitur versorem $n$ superficiei materialis $\left(^{3}\right.$ ) (utrum haec superficies paries in quiete maneat an secus) dirigi, ut sit

$$
D n=\delta_{n}+W n
$$

In pariete quieto igitur

$$
D_{n}=\delta_{n}-\frac{1}{2} w f
$$

At cum sit

$$
\boldsymbol{D} \boldsymbol{n}=D_{11} \boldsymbol{n}+D_{12 \boldsymbol{e}}+D_{13} \boldsymbol{f}
$$

sequitur esse $D_{11}=\delta, D_{12}=D_{21}=0, D_{13}=D_{31}=-\frac{1}{2} w$. Quoniam autem tractus secundum omnia in plano versori $n$ normali directa nulli, $D_{22}=D_{23}=$ $=D_{33}=0$, ut eveniat

$$
\begin{aligned}
\boldsymbol{D} & =\delta_{\boldsymbol{n}} \otimes \boldsymbol{n}-\frac{1}{2} w(\boldsymbol{n} \otimes \boldsymbol{f}+\boldsymbol{f} \otimes \boldsymbol{n}) \\
& =\delta_{\boldsymbol{n}} \otimes \boldsymbol{n}+\boldsymbol{n} \otimes(\boldsymbol{W} \boldsymbol{n})+(\boldsymbol{W} \boldsymbol{n}) \otimes \boldsymbol{n},
\end{aligned}
$$

quod theorema elegans primus dedit D. Bhutus Caswelutus $(9$ ) cuius demonstratio in transformatione KELVINII fundata est.

ut eveniat

et

$$
\begin{gathered}
\operatorname{grad} \dot{x}=D+W \\
W e=0, \quad|e|=1 .
\end{gathered}
$$

(7) V. ex. gr, Aeq. (76.6) tractatus nostri de theoriis elassicis camporum. Aeq. (22) primum a $D^{0}$. RATIB BERKER inventa est, qui eam auxilio transformationis KeLviNI deduxit. V. schedulam eius "Sur certaines propviétés de l'effort qui s'exerce sur une parois en contact avec un fluide visqueux», C.R. Acad. Sci. Paris 232, 148-149 (1951).

(8) Superficies in motu fluidi o materialis. denominatur, si particula fluidi in ea superficie reperitur ant semper ant numquam.

(9) V. eius commentarium, "Kinematics and stress on a surface of rest", Arch. Rational Mech, Anal. 26, 385.399 (1967). 
Ex Aeq. (25) perspicitur versorem $n$ superficiei parallelum directioni principali tensoris $\boldsymbol{D}$ numquam dirigi posse, nisi sit $w=0$. Calculo facili inveniuntur tractus principales $D_{i}$ :

$$
\begin{aligned}
2 D_{1} & =\delta+\sqrt{\delta^{2}+w^{2}} \geqq 0, \\
D_{2} & =D_{(\mathrm{e})}=0, \\
2 D_{3} & =\delta-\sqrt{\delta^{2}+w^{2}} \leqq 0 .
\end{aligned}
$$

Insuper est $D e=0$; axis igitur circumactuum simnl axis principalis tractuum, et insuper illa quidem axis secundum quam evanescit tractus. Angulus $\varphi$ inter versorem $n$ superficiei et directionem in quam tractus maximus fit, ex aequatione

$$
\tan 2 \varphi=\frac{w}{\delta}
$$

petendus est. Quare

$$
\begin{aligned}
& \varphi=0 \quad \text { si } w=0, \quad \delta>0 ; \\
& 0<\varphi<\frac{1}{4} \pi \text { si } w \neq 0, \quad \delta>0 ; \\
& \varphi=\frac{1}{4} \pi \quad \text { si } w \neq 0, \quad \delta=0 ; \\
& \frac{1}{4} \pi<\varphi<\frac{1}{2} \pi \text { si } w \neq 0, \quad \delta<0 ; \\
& \varphi=\frac{1}{2} \pi \text { si } w=0, \quad \delta<0 .
\end{aligned}
$$

Numerum vorticalem $\mathfrak{⿰}$, qui definitione

$$
\begin{aligned}
\mathfrak{w a} & =\frac{|W|}{|D|}=\sqrt{\frac{\operatorname{tr} W W^{T}}{\operatorname{tr} D D^{T}}}=\sqrt{\frac{-\operatorname{tr} W^{2}}{\operatorname{tr} D^{2}}}, \\
& =\frac{w}{\sqrt{2} \sqrt{D_{1}^{2}+D_{2}^{2}+D_{3}^{2}}}
\end{aligned}
$$

introducitur, calculus facilis prodit:

$$
\mathfrak{w}=\frac{w}{\sqrt{w^{2}+2 \delta^{2}}} .
$$

Quam ob rem in puncto parietis quieti

$$
\mathfrak{w a}<1
$$


nisi motus ibi isochoricus sit, at in motu isochorico semper et ubique in pariete quieto

$$
\mathfrak{w a}=1
$$

nisi sit $w=0$.

\section{5. - Applicatio numeri tractilis in puncto parietis quieti.}

Ut in paragrapho superiori vidimus, versor $n$ superficiei in quiete in directionem principalem tractus non cadat nisi ibi sit $v=0$. Cum in motu fluidi viscosi directiones principales conatus eaedem ae illae tractus sint, et cum directio tractus maximi eadem ac directio pressionis minimae sit, sequitur

Treorema. - In punclo parielis quieti in motu fuidi viscosi pressio normalis in superficie semper maior est pressionibus, quas patiuntur aliqua elementa superficiei obliqua, nisi casu $w=0$.

Si Te $<1$, pressio in superficie sane affirmativa, sed ex theoremate sequitur hanc pressionem etiam si sit $\mathbb{T} e \geqq 1$ affirmativam evenire posse. Si pressio negativa nt indicium separationis tenenda est, videtur fluidum in puncto parietis ubi modo $T e=1$ plus a se quam a pariete abscedere conari.

Angulus $\theta$ quem prodit Aeq. (27) fit in motu fluidi viscosi angulus inter versorem $n$ parietis et versorem elementi quod pressionem normalem minimam patitur. Ex Inaeq. (28) colligitur inter alia illum angulum in motu dilatandi angulum semirectum superare non quire. Si modo dilatatio praemagna, erit $\varphi=0$ quam proxime, id est, in expansione vehementi erit $p_{(n)} \approx p_{\min }$, quae conjunctio ex Aeq. (36) quoque percipitur.

Ut haec omnia olarius perspiciantur, opus est formulas quasdam consignare, quae se statim ex rebus supra traditis offeruntur. Ex Aeqq. (6) et (22) sequitur theorema elegans amici illustris RATIB Berker $\left({ }^{10}\right)$ de tractione $t$ quae ad parietem quietum adnititur:

$$
t=[-p+(\lambda+2 \mu) \delta] n-\mu w f,
$$

quam ob rem pressio normalis $p_{(n)}$ sequenti formula datur:

$$
p_{(n)}=p-(\lambda+2 \mu) \delta .
$$

Pressio autem normalis minima ex superioribus Aeqq. (8) et (26) invenitur

$$
p_{\min }=p-\lambda \delta-\mu\left(\delta+\sqrt{\delta^{2}+w^{2}}\right) .
$$

(10) Op. eit. in Adnotatione 7. 
Hine

$$
p_{(n)}-p_{\min }=\mu\left[\sqrt{\delta^{2}+w^{2}}-\delta\right],
$$

quae differentia semper affirmativa est, nisi $v=0$. Condicio igitur $\tau e>0$ abunde sufficit, at in superficie quieta pressio negativa non detur.

Vulgo scitur ex Inaeqq. (2) $)_{2,3}$ sequi inaequalitatem $\lambda+2 \mu>0$. Quam ob rem ex Aeq. (34) colligitur esse

$$
p_{(\boldsymbol{n})} \lesseqgtr p \quad \text { si } \quad \delta \gtreqless 0 .
$$

Ergo, $p_{\min }<p$ si $\delta>0$, id est, in motu dilatandi. Quaeretur autem si pressio normalis minima semper infra pressionem staticam jacere dabeat etiam in motn astringendi. Ex Aeqq. (35) et (4) reperitur esse

$$
\frac{3\left(p-p_{\min }\right)}{\mu}=(2 \mathfrak{J B}+1) \delta+3 \sqrt{\delta^{2}+w^{2}} .
$$

Analysis facilis, quam tnto lectori relinqui licet, prodit

Theorema. - In puncto parietis quieti, posita vorticitate $w+0$, erit semper

$$
p_{\min }<p \text { si aut } \delta \geqq 0 \text { ant } \mathfrak{S B} \leqq 1 .
$$

Si autem $\mathfrak{B B}>1$ et $\delta<0$, erit

$$
p_{\min } \lesseqgtr p \quad \text { si }-\delta \leqq \frac{3 w}{2 \sqrt{(\sqrt{B}-1)(\sqrt{B}+2)}} .
$$

Pressio statica $p$ igitur ad indicium pressionum normalium verarum longe uon sufficit. In motu dilatandi flnidi cuiuscunque et in omni motu fluidi in quo $\mathfrak{B} \leqq 1$, elementa dantur in puncto parietis quae pressiones normales pressione statica minores patiuntur; ex contrario si $J 6>1$, in motu astringendi satis vehementi, omne elementum in pariete pressionem normalem patitur, quae pressionem staticam superat.

In puncto parietis auxilio Aeqq. (26) formae simpliciores numerorum Te et $\mathrm{Tr}$ Aeqq. (9) ef (13) definitorum reperiuntur:

$$
\begin{aligned}
& \tau_{e}=\frac{\mu}{p}\left[\frac{1}{3}(1+2 \mathfrak{B}) \delta+\sqrt{\delta^{2}+w^{2}}\right], \\
& \tau \mathfrak{r}=\frac{\mu}{p} \sqrt{2 \delta^{2}+w^{2}} .
\end{aligned}
$$


Plane

$$
\mathbb{T e} \leqq\left(1+\frac{1+2 \mathfrak{B}}{3 \sqrt{2}}\right) \mathbb{C}^{\mathfrak{t}}
$$

\section{6. - Numerus tractilis in motu fluidi nullius compressionis capacis.}

Si fluidnm nullius compressionis capax, non solum $\delta=0$ set etiam pressio statica $p$ statu cinematico fluidi non determinatur, at potius variabilis soluta manet. In aequationibus differentialibus pressio constans quaevis (aut functio solius temporis) $p_{0}$ omni solutioni adjungi potest, ad quam determinandam opus est (ex. gr.) pressionem staticam in aliquo puncto assignare. Ex Aeq. (7) patefit pressio normalis quam patitur elementum superficiei cuius versor $n$ :

$$
p_{(n)}=p-2 \mu D_{(n)}
$$

Quia in mota isochorico et non rigido $D_{1}>0$,

$$
\min p_{(n)}<p
$$

Ergo, ut omnis pressio normalis nonnegativa sit, necesse est pressionem staticam affirmativam esse,

$$
p>0
$$

motu rigido seposito.

His intellectis, possumus ut ante progreải et, Aeq. (13) revisa, numerum tractilem formula definire, quae in mota isochorico gasi valet, id est

$$
\tau e \equiv \frac{2 \mu D_{1}}{p}
$$

Ex analysi supra tradita colligitur

Theonema. - Ot in motu nonrigido fluidi viscosi nullius compressionis capacis nullum elementum superficiei pressionem normalem negativam sustineat, necesse est et sufficit esse

$$
p>0, \quad T_{e}<1
$$

Calculo facili invenitur limes

$$
\mathbb{T e} \leqq \frac{2}{\sqrt{3}} \mathbb{C} \mathfrak{r}
$$


ubi aequalitas valet si $D_{2}=D_{3}=-\frac{1}{2} D_{1}$, ubi iam ex Aeqq. (18) et (19) in casu gasi modo STokesiano obtemperantis videtur.

In puncto parietis quieto ex Aeqq. (26) elicitur ut sint

$$
D_{1}=\frac{1}{2} w, \quad D_{2}=0, \quad D_{3}=-\frac{1}{2} w
$$

ex Aeq. (32)

$$
\mathfrak{W} \mathfrak{W}=1
$$

ex Aeqq. (36) et (38)

$$
p_{(n)}-p_{\min }=p-p_{\min }=\mu w ;
$$

ex Aeqq. (41)

$$
\mathfrak{T e}=\tau_{\mathfrak{r}}=\frac{\mu w}{p}=\frac{p_{(n)}-p_{\min }}{p}
$$

ex Aeq. (27)

$$
\varphi=\frac{1}{4} \pi
$$

\section{7. - De pressionibus negativis in regione.}

Adhuc de puncto uno solo tractatum est. In regione quam fluidum mo. vens implet, numerus tractilis $\mathbb{C} e$ campus scalaris fit. In regione clausa assumit hic campus in aliquo puncto valorem maximum $\tau e_{\max }$. Ut pressiones negativae in tota regione desint, necesse est et sufficit limes

$$
\widetilde{T} \mathfrak{e}_{\max }<1 .
$$

Difficilia inventu et demonstratu erunt theoremata generalia quae punctum locent ubi valor maximus praesit. Theoremata talia vere de pressione statica $p$ cognoscuntur, quae in casibus generalioribus praestant ut loous pressionis minimae alicubi in finibus semper reperiendus sit ${ }^{(1)}$. Habitus autem autovalorum $D_{i}$ in regione non adhuc indagatus esse videtur.

(11) Theoremata huiusmodi generalissima demonstravi in commentatione "Tro measures of vorticity", J. Rational Mech. Anal. 2, $178-217$ (1953). V. Corollarium Theorematis 8. Ex. gr., si in regione aperta a fluido viscoso nullius compressionis capaci impleta sit $\mathfrak{W} \leqq 1$, pressio statica $p$ minima non datur. 
Pro flaidis nullius compressionis capacibus tamen observatio simplicior notanda est. Secundum theoriam differentia $p-p_{0}$, ubi $p_{0}$ functionem solius temporis $t$ denotat, ex campo velocitatum determinatur, qui finibus datis et velocitatibus incohantibus datis convenit, sed postea quantitas $p_{0}$ libera electu manet. Sit igitur una cum determinatione pressionis $p$ staticae valor maximus numeri tractilis in data regione clansa $\mathcal{C E}_{\max }^{(1)}$. Si pressioni $p$ con. stans $p_{0}$ adjunota erit, ut vere de supra dictis licet, invenietur ex Aeq. (52) de numero tractili $\widetilde{T e}$, qui novae solutioni convenit,

$$
\begin{aligned}
\widetilde{T e} & =\frac{2 \mu D_{1}}{p} \cdot \frac{p}{p+p_{0}}, \\
& \leq \widetilde{T} e_{\max } \cdot \frac{1}{1+p_{0} / p_{\max }},
\end{aligned}
$$

ubi $p_{\max }$ valorem maximum pressionis staticae in solutione anteriori denoteret. Ut in nova solntione, ex anteriori adjunctione constantis pressionis $p_{0}$ orta, pressiones normales negativae desint, sufficit eligere talem $p_{0}$ at sit

$$
\frac{p_{0}}{p_{\max }}>\mathbb{T} e_{\max }-1
$$

Haec ratio prodit

Theonma. - Data solutione equationum Navier-Stokesianarum in regione clausa, semper licet adjungere campo p pressionis staticae constantem $p_{0}$ talem ut eveniat solutio altera in qua pressiones negativae desint.

Indagationem, quem hie exposui, munere adjuvit U.S. National Science Foundation. Amico Piof. Jak. Poultney gratias ob consilia ago.

d. 1. ante Kal. Jan. 1970. 